\title{
Phase IV Clinical Trials of Biosimilars for Cancer: Extrapolation of the 'Basket Concept' of Trials
}

\author{
Jayesh Bhatt*, Manjunath Krishnappa, Deven Parmar \\ Cadila Healthcare Ltd., Zydus Research Centre, Sarkhej-Bavla N.H. No. 8A, Moraiya, Ahmedabad, Gujarat, INDIA.
}

Of late, the concept of 'basket trials', testing the effect of a single drug in multiple indications is receiving increased attention. ${ }^{[1]}$ Can this concept be extrapolated to the clinical development of biosimilars? Majority of the regulatory agencies have the provision for the approval of biosimilars for one or more additional indications based on the concept of extrapolation without necessarily conducting trials in those indications..$^{[2-4]}$

However, such biosimilars that have been granted marketing approval based only on the extrapolation of data and not from the evidence of clinical trials in those indications may not provide the required confidence to the prescribers and patients alike. Furthermore, extrapolation of the immunogenicity findings to a different population may have its own limitations.

This is where the 'Basket trial concept' can help the developers to fill up the gaps in the required data and provide the much needed confidence. However, this concept possibly cannot be used at the phase $2 / 3$ stage since normally trials are conducted in just one indication. Studies in different indications will add considerable cost to the developers.

The optimal juncture to use this concept will be at the post-marketing/ Phase 4 stage. One single structured phase 4 study or a patient registry can be conducted possibly involving all the approved indications. Safety, efficacy and long term immunogenicity can be the outcome measures. The sample size can be determined based upon the operational feasibility and the analysis and the results can be stratified indication wise.
In summary, the use of the 'Basket concept' will provide the much needed confidence to the regulators, prescribers and the patients and also help the drug developers to contain the overall clinical development costs of the biosimilars.

\section{REFERENCES}

1. Kristen MC, Mithat G, Ronglai S, David MH, Gregory JR, Colin BB et al. Basket Trials in Oncology: A Trade-Off between Complexity and Efficiency. Journal of Clinical Oncology. 2017;35(3):271-3.

2. Guideline on similar biological medicinal products. London, UK: Committee for Medicinal Products for Human Use (CHMP), European Medicines Agency. 2014. [Accessed July 22, 2018].

3. United States Food and Drug Administration. Scientific considerations in demonstrating Biosimilarity to a reference product: guidance for industry. Silver Spring, MD: US Department of Health and Human Services, Center for Drug Evaluation and Research (CDER), Center for Biologics Evaluation and Research (CBER). 2015. [Accessed July 22, 2018].

4. Guidelines on evaluation of similar biotherapeutic products (SBPs) Geneva, Switzerland: World Health Organization Expert Committee on Biological Standardization. 2009. [Accessed July 22, 2018]

Correspondence to:

Dr. Jayesh Bhatt, PhD, Email: jkbhatt@gmail.com

Cite this article as: Bhatt $\mathrm{J}$, Manjunath K, Parmar D. Phase IV Clinical Trials of Biosimilars for Cancer: Extrapolation of the 'Basket Concept' of Trials. J Pharm Pract Community Med. 2018;4(3):131. 